\title{
Multimodal Inference for Driver-Vehicle Interaction
}

\author{
Tevfik Metin Sezgin \\ Computer Laboratory \\ University of Cambridge \\ Cambridge, CB3 0FD, UK \\ Metin.Sezgin@cl.cam.ac.uk
}

\author{
Ian Davies \\ Computer Laboratory \\ University of Cambridge \\ Cambridge, CB3 0FD, UK \\ Ian.Davies@cl.cam.ac.uk
}

\author{
Peter Robinson \\ Computer Laboratory \\ University of Cambridge \\ Cambridge, CB3 0FD, UK \\ Peter.Robinson@cl.cam.ac.uk
}

\begin{abstract}
In this paper we present a novel system for driver-vehicle interaction which combines speech recognition with facialexpression recognition to increase intention recognition accuracy in the presence of engine- and road-noise. Our system would allow drivers to interact with in-car devices such as satellite navigation and other telematic or control systems. We describe a pilot study and experiment in which we tested the system, and show that multimodal fusion of speech and facial expression recognition provides higher accuracy than either would do alone.
\end{abstract}

\section{Author Keywords}

Driver monitoring, facial-expression recognition, speech recognition, multimodal inference.

\section{ACM Classification Keywords}

H5.2 User Interfaces

\section{INTRODUCTION}

Accurate measurement of drivers' intentions and responses is an important requirement for effective human-vehicle interaction. Detecting user response reliably is especially important in interaction scenarios where feedback is expected in response to a question (e.g., posed by an in-car navigation system). So far, in-car interaction modalities have been restricted to traditional graphical dialog-box representations and speech-based input. Traditional graphical representations usually require interacting with small touch-sensitive displays, and can be distracting because of the visual attention required for the interaction. Speech-based interfaces, on the other hand, offer a more natural modality for interaction, although their usefulness is subject to a number of limitations.

We have developed a framework for automatic analysis of drivers' facial expressions with the goal of adding facial displays to the list of modalities available for humanvehicle interaction. Specifically, we have investigated the feasibility of combining head-based displays with speech in order to achieve higher recognition results in the presence of noise. We studied the effects of noise in an interaction scenario that required responses to a series of "yes/no" questions, which are typical in interacting with a navigation system (e.g. "The gas is running low. Would you like directions to the nearest gas station?").
Because vehicle-noise and the willingness of the driver to express themselves clearly through spoken dialogue are the primary causes of misrecognized speech, we focused our investigation on intelligent fusion of head-display and speech information for varying noise levels and varying speaker volumes. Using our in-house driving simulator, we conducted a pilot study where we recorded a participant answering a series of "yes/no" questions while driving. We recorded separate audio and video streams that captured the driver's speech and facial displays. Based on promising results from the pilot study, we conducted a larger controlled experiment with 4 further subjects (age 22-50) to verify our findings.

We implemented a speech recognition application for processing the audio and used our own facial expression recognition software [5] to interpret the video stream. We used Support Vector Machines to fuse audio- and videobased inference results and constructed a multimodal recognition engine that outperforms the individual modalities.

\section{EXPERIMENTAL SETUP}

Figure 1 shows the physical setup of our experiment. We had a driving simulator equipped with a digital video camera for recording facial displays (1). A microphone secured to the frame in front of the driver (2) was used to record audio for speech recognition. Two pairs of speakers

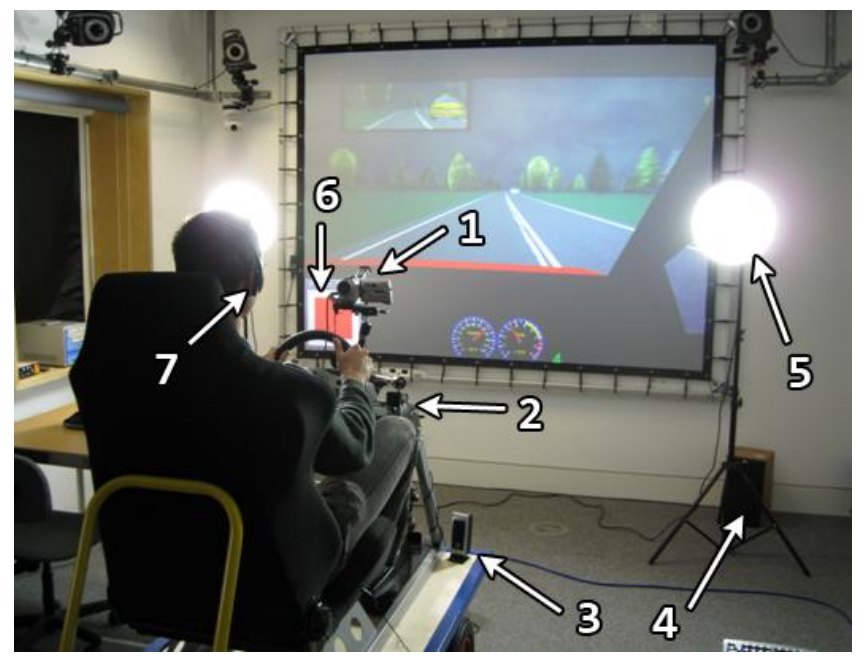

Figure 1: The physical setup of our experiment 


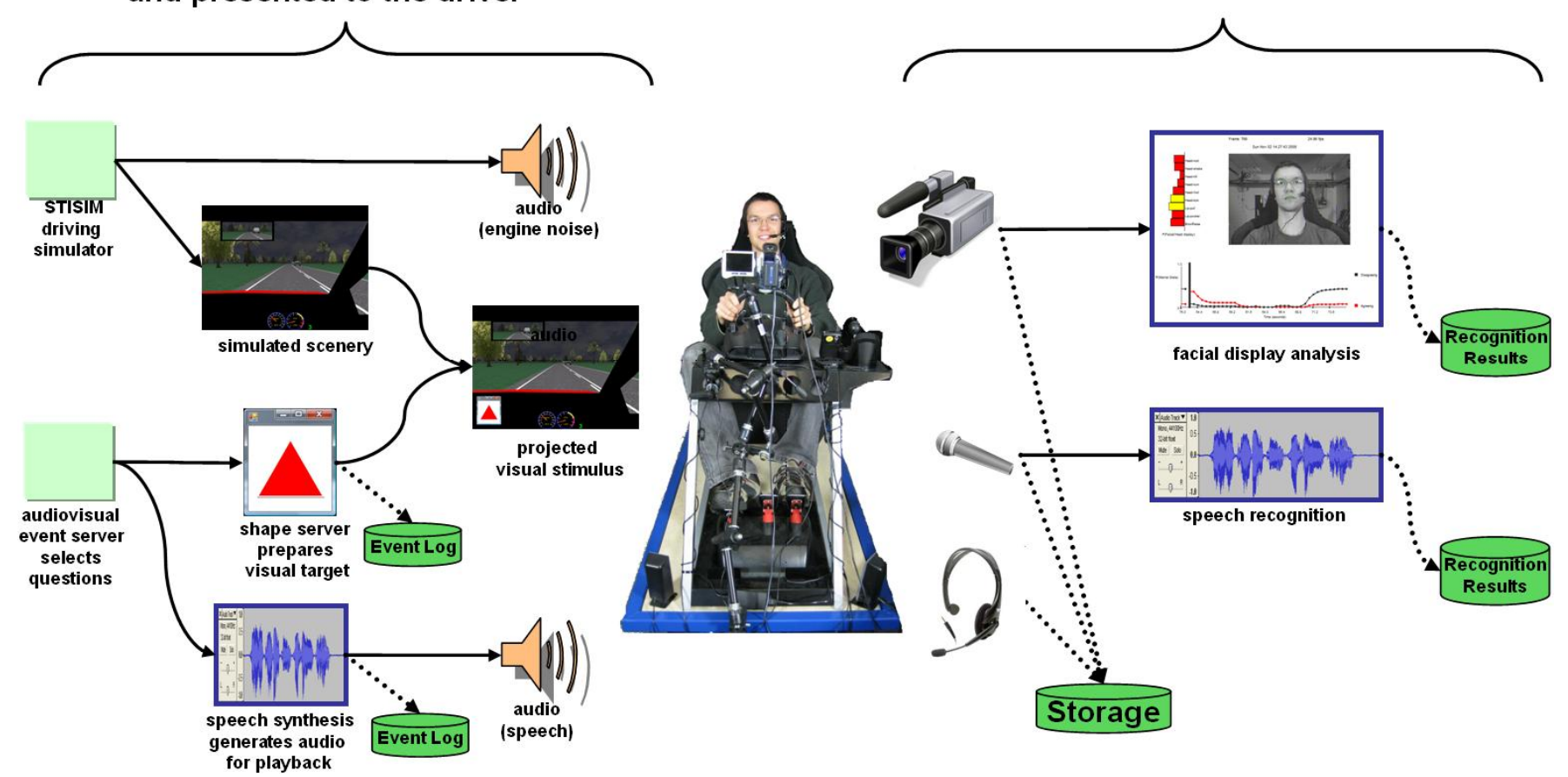

Figure 2: System Structure

were used for playing audio stimuli consisting of questions for the driver (3), and simulated engine noise (based on vehicle speed and road conditions) generated by the STISIM driving simulator software (4). A pair of studio lights (5) was used to control the lighting conditions. During the experiment, the driver was asked a series of questions referring to a target shape displayed next to the virtual dashboard projected on a screen (6). The driver also wore a headset with microphone to record higher quality speech for further analysis (7).

The pilot study was designed to investigate the effects of noise in an interaction scenario requiring responses to a series of "yes/no" questions. Such situations frequently arise while interacting with a navigation system. In order to elicit a verbal or non-verbal agreement/disagreement response, we designed a task where the user is first presented a geometric shape (like the red triangle as shown in Figure 2), and then asked a question about the shape, which can be answered verbally ("yes" or "no"), and nonverbally (head-nod or head-shake). We refer to each round of shape presentation as a trial. In order to collect representative data for varying noise levels, we had a total of 60 trials consisting of 30 questions requiring an affirmative response, and 30 requiring a negative response. For the subsequent larger experiment, we ran 100 trials with each of the 4 participants. The order of trials was randomized. Each trial consisted of playing an audio clip which asked a question (e.g. "Is the shape a red triangle?"), displaying the target shape in a particular colour for 2 seconds, then expecting an appropriate verbal and/or nonverbal response from the driver. We waited 5 seconds between trials.
The primary source of noise in the study was the engine noise generated by the STISIM driving simulator based on the vehicle speed and road conditions. We started the experiment with the noise volume set to zero, and gradually increased the noise level over the course of the drive. The loudest noise level was $75 \mathrm{dBA}$ - slightly above that experienced by the driver of a transit van at about $60 \mathrm{mph}$ (100kph). For the final 40 trials in the large controlled experiment, the driver was asked to speak louder or quieter in order to provide additional data relating to variation in speaker loudness.

The audio stream used for speech recognition was captured by a microphone on the dashboard (\#2 in Figure 1). The speech was processed by a Microsoft SAPI 5 application customized to recognize 29 words that a driver would be likely to use in the context of driving including "yes," "no," and five variants (such as "yeah", "nope"). The video used for recognizing head displays was captured by a digital camcorder positioned directly in front of the driver (\#1 in Figure 1) and was processed by our mind-reading software [5] trained to recognize facial displays for agreement and disagreement.

\section{RESULTS}

\section{Audio-Based Recognition Results}

Speech recognition events were attributed to particular questions by nearest-neighbour matching. Speech events in the audio were identified by clustering. The RMS value of the audio data for each cluster provided a measure of speaker loudness. The background noise was measured by averaging the absolute value of the audio signal in the time between the question finishing and the driver speaking. 
Figure 3 presents speech recognition results from the pilot study for increasing levels of noise. As seen in this graph, speech recognition works reasonably well when the noise is low. The recognition accuracy is very poor for high noise levels, and there is a transitional gray zone between the high-noise and low-noise segments where the recognition results are unpredictable. The recognition accuracy based on speech alone was $57 \%$ in the pilot study.

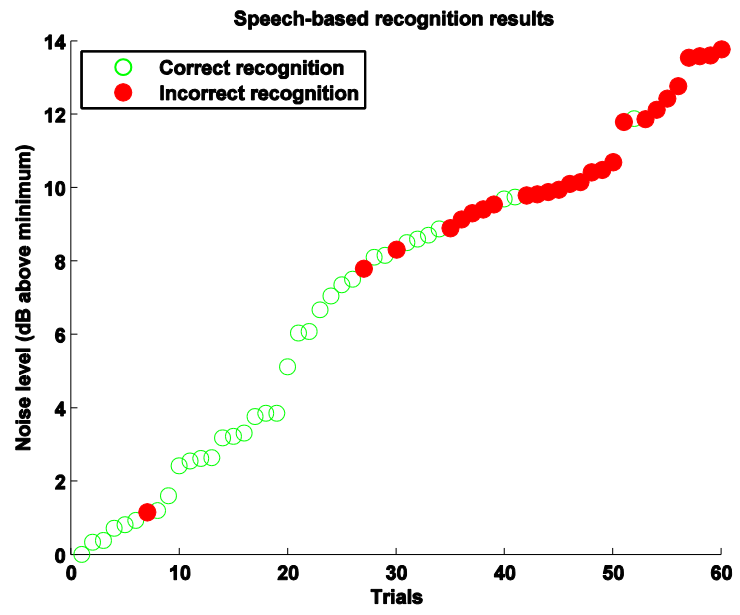

Figure 3: Speech-Based Recognition Results

\section{Video-Based Recognition Results}

To compute the head display hypotheses for each trial, we compared the average probabilities of agreement and disagreement for a short video segment taken briefly after the completion of the question. Although our facial display analysis software computes probability scores for agreement and disagreement, these do not represent actual probabilities in the Bayesian sense and this prohibits direct comparison of the values. Therefore we treat these numbers as scores and train a linear SVM classifier that maps a pair of agreement/disagreement scores to an agreement or disagreement decision. Figure 4 shows the video-based recognition results from the pilot study for increasing levels

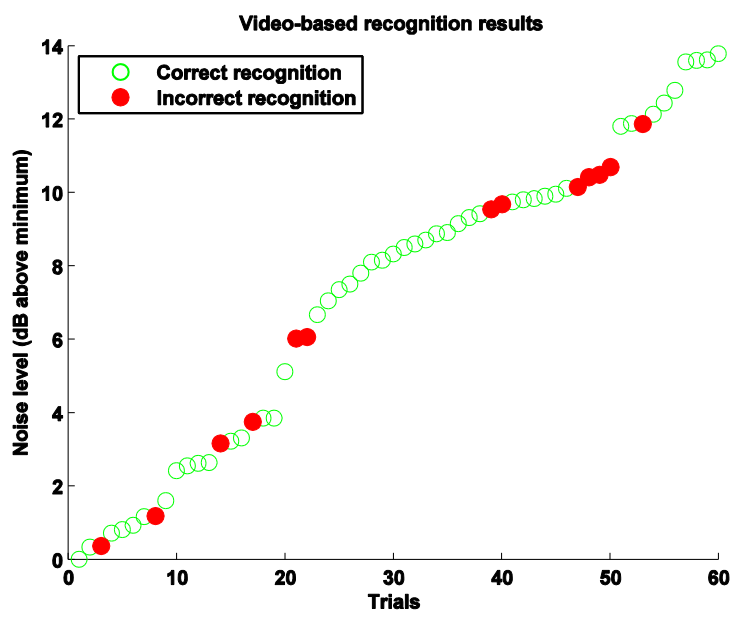

Figure 4: Video-Based Recognition Results of noise. As seen in this graph, video recognition generally works well, and its accuracy does not appear to depend on the noise level. The recognition accuracy based on video alone was $78 \%$ in the pilot study.

\section{Multimodal Fusion Results}

Our framework for multimodal fusion is based on the observation that speech recognition works remarkably well for low-noise conditions, but performs quite badly in high noise conditions, while the video-based recognition performance is reasonably accurate regardless of the noise level. We fuse the audio and video information by treating the results of our speech and head-display analysis as inputs to a classifier along with the noise level of the environment. More specifically we consider a classification problem where the inputs are 3-tuples $\left\langle a_{i}, v_{i}, n_{i}\right\rangle$, which respectively represent the class assigned by the speech recognizers $\left(a_{i}\right.$ : yes/no/other), head-display recognizers ( $v_{i}$ : agreement/ disagreement), and the noise level $\left(n_{i}\right)$ for trial $i$. For the subsequent experiment, we also included speaker loudness. Although this appears to be a simple construction, the high dimensional space representing the decision problem is sufficiently complex and is not linearly separable. Although some of the categorical data inputs could be re-ordered to achieve a better space, it is highly likely that the nonlinearity would still not be avoided when more features (such as the complexity of the speech recognition grammar) were added to the input space. In order to deal with this non-linearity, we trained Support Vector Machines (SVMs) with Radial Basis Function kernels for multimodal fusion.

We measured the performance of our SVM using 10-fold cross validation, and ran it 10 times while randomly shuffling the training and testing sets, thus performing bootstrapping. Representative results of one cross-validated run are shown in Figure 5. The average recognition rate for the multimodal classifier was $89 \%$ in the pilot study, with a standard deviation of 3.1. This is substantially higher than either of the individual modalities considered separately.

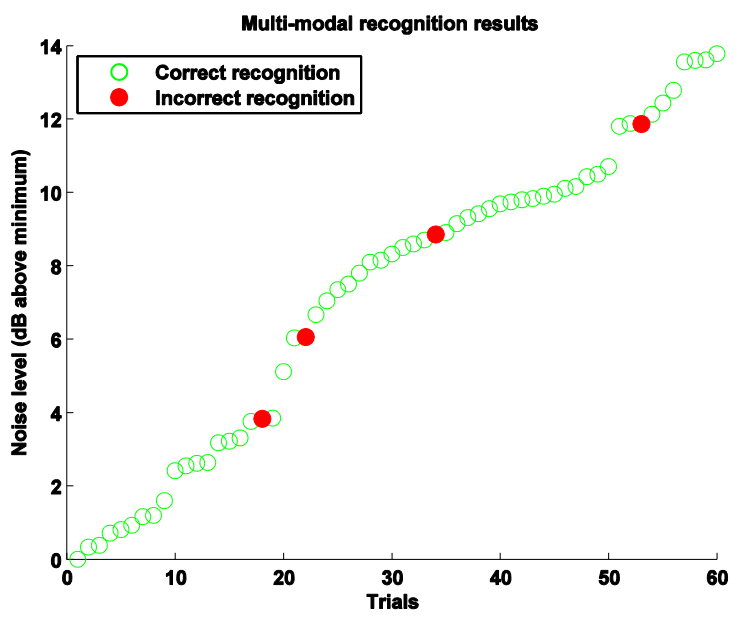

Figure 5: Multi-Modal Recognition Results 
The results obtained in the larger controlled experiment (summarised in Table 1) supported those from the pilot study. In all cases multimodal inference outperformed each individual mode. Note that our system was particularly valuable for subject 4 , for whom speech recognition alone was entirely inadequate.

\begin{tabular}{|c|c|c|c|c|}
\hline Subject & $\begin{array}{c}\text { Speech-Based } \\
\text { Recognition } \\
\text { Accuracy (\%) }\end{array}$ & $\begin{array}{c}\text { Video-Based } \\
\text { Recognition } \\
\text { Accuracy (\%) }\end{array}$ & $\begin{array}{c}\text { Multimodal } \\
\text { Accuracy } \\
\text { (Mean, \%) }\end{array}$ & $\begin{array}{c}\text { Multimodal } \\
\text { Accuracy } \\
\text { (S.D.) }\end{array}$ \\
\hline 1 (Pilot) & 57 & 78 & 88.7 & 3.1 \\
\hline 2 & 46 & 67 & 72.3 & 2.5 \\
\hline 3 & 54 & 69 & 75.8 & 1.5 \\
\hline 4 & 31 & 65 & 70.4 & 3.4 \\
\hline 5 & 39 & 69 & 73.9 & 2.6 \\
\hline
\end{tabular}

Table 1: Controlled Experiment Recognition Results

\section{DISCUSSION}

These results suggest that multimodal approaches where the individual modalities complement each other and compensate for their shortcomings have the potential to reduce recognition errors, substantially improve drivervehicle interaction, and enhance the overall driving experience. Recognition accuracy for each individual system could easily be increased with further refinement, but it seems likely that multimodal fusion would always yield a better result.

\section{RELATED WORK}

It has long been recognised that current driver-vehicle interaction techniques are inadequate for safe and effective use of increasingly complex in-car devices. People have begun to investigate alternative methods, such as speech recognition [1]. A considerable amount of work has been done to tackle the problem of speech recognition in noisy environments [2,3], with good results. However, recognition accuracy always decreases as noise increases and there is a limit to how much it can be improved.

Automated facial expression recognition has also been dealt with previously [4,5], and explicit head-nod and shake detection has been implemented [6]. Although this experiment only deals with agreement and disagreement, our facial expression recognition software [5] uses more than just nod and shake detection and is capable of distinguishing several more mental states.

Several people have combined vision-based approaches with speech recognition - usually in the context of broader affective inference for emotion recognition [8,9]. Work has also been done on supporting speech recognition specifically [7] and on combining vision and speech in automotive environments for identification and authentication [10].

\section{FUTURE WORK}

These results suggest that it would be worth pursuing further investigations of affective inference as a component in the dialogue between a driver and an in-car telematic system.

The next step would be to move from detection of simple agreement and disagreement to a more elaborate dialogue involving a broader range of options in a larger and more realistic task. This could also involve understanding the level of the driver's concentration so as to avoid distraction from more critical driving tasks.

\section{SUMMARY}

We have demonstrated, tested and validated a system for driver-vehicle interaction which uses multimodal fusion of speech and facial expression recognition. We have shown that combining these inference techniques gives a level accuracy unattainable when using either system on its own. The architecture of the inference system we built provides a more general framework in which new techniques can be tested.

\section{REFERENCES}

1. Lee, J.D. et al. Speech-based Interaction with In-vehicle Computers: The Effect of Speech-based E-mail on Drivers' Attention to the Roadway. Human Factors 43, 2001, 631-640.

2. Gong, Y. Speech recognition in noisy environments: A survey. Speech Communication, 16 (1995), 261-291.

3. Frey, B.J. et al. Learning dynamic noise models from noisy speech for robust speech recognition. In NIPS, 2002.

4. Fasel, B., Luettin, J. Automatic facial expression analysis: a survey. Pattern Recognition 36, 1 (2003), 259-275.

5. El Kaliouby, R., Robinson, P. Real-Time Inference of Complex Mental States from Facial Expressions and Head Gestures. In Real-Time Vision for HumanComputer Interaction, 2005, 181-200.

6. Rong, G., Tan, W. A real-time head nod and shake detector using HMMs. Expert Systems with Applications 25, 3 (2003), 461-466.

7. Cooke, M. et al. An audio-visual corpus for speech perception and automatic speech recognition. The Journal of the Acoustical Society of America 120, 5 (2006) , 2421-2424.

8. Busso, C. et al. Analysis of emotion recognition using facial expressions, speech and multimodal information. Proc. ICMI 2004, 205-211.

9. Jaimes, A., Sebe, N. Multimodal human-computer interaction: A survey. Computer Vision and Image Understanding 108, 1-2 (2007), 116-134.

10.Erzin, E. et al. Joint Audio-Video Processing For Robust Biometric Speaker Identification In Car. DSP for In-Vehicle and Mobile Systems, 2005. 237-256 